\title{
Tecnologías de Fabricación Digital y Biomateriales
}

\section{Biomaterials and Digital Manufacturing Technologies}

Presentación: 6/10/2020

\section{Doctorando:}

\section{Camila M. Picco}

Instituto de Investigaciones en Catálisis y Petroquímica, Facultad de Ingeniería Química, Universidad Nacional del Litoral - Consejo Nacional de Investigaciones Científicas y Técnicas (CONICET)- Argentina.

cami.picco38@gmail.com

Director/a:

\section{Silvina Regenhardt}

\section{Co-director/a:}

\section{Nancy Balsamo, Santiago Palma}

\section{Resumen}

Las primeras tres revoluciones industriales tenían la característica de que se desarrollaron en momentos de la historia donde se consideraba que los recursos naturales eran ilimitados y que la producción masiva no generaría consecuencias sobre medio ambiente y las personas. Sin embargo, en los últimos años se ha demostrado el grave impacto que ha tenido la actividad humana sobre el medio ambiente. Es por esto se han estado sentado las bases para la cuarta revolución industrial, en donde se plantea un modelo productivo, social y económico que cambie de manera innovadora, eficiente y sustentable el actual sistema.

Los autores Jose Luis del Val (2016); klaus schwab (2016); Joao Saint-Aubyn (2016), entre otros, caracterizan la cuarta revolución industrial o industria 4.0 es un nuevo paradigma que pretende dar un salto cualitativo a través de la evolución y conexión de herramientas y recursos, en busca de conformar las llamadas 'Fábricas inteligentes'. Este modelo pretende dar competitividad a la industria a través de la producción personalizada y el diseño. Se busca vincular los sistemas digitales, físicos y biológicos para optimizar el bienestar humano y del medio ambiente. Pretende orientar la producción hacia una economía circular, donde el principal objetivos es satisfacer las necesidades de los usuarios, y generar un cambio de paradigma en la producción, reduciendo los desechos y utilizando energías renovables. Asimismo, esta revolución acompaña un nuevo modelo denominado economía circular, el cual se interrelaciona con la sostenibilidad, cuyo objetivo principal es mantener en circulación durante el mayor tiempo posible los productos, materiales y recursos, reduciendo la producción de residuos. Se trata de implementar un sistema circular, no lineal, basado en el principio de cerrar el ciclo de vida de los productos. Dentro de esta revolución el diseño cumple un rol fundamental para lograr el fin esperado. Es necesario desarrollar un sistema diseñado que permita la inserción de esta revolución al medio actual facilitando la comprensión y los resultados. A este escenario circular se incorpora la biofabricación, la aplicación de la biotecnología para cultivar y hacer crecer los objetos en lugar de manufacturarlos. Esta manera de producir crea un cambio de paradigma, ya que la bioproducción responde a la Economía Circular, en donde la basura no existe ya que los residuos se reciclan y se transforman y, al ser biodegradables, vuelven a su origen. 
Es en esta nueva concepción de fabricación a través del cultivo de materiales en donde nos posicionamos en la investigación con el objetivo de buscar soluciones a través del diseño, la biofabricación y el uso de tecnologías de fabricación digital. Para esto se propone un plan de trabajo y uso de metodologías para llegar a resultados a través de la validación practica que permitan introducir una innovación al sistema productivo actual. Se propone generar diseños que se introduzcan al sistema actual, reemplacen materiales plásticos como el poliestireno expandido (tergopol) y colaboren con el medio ambiente bajo un modelo de economía circular, es decir, aprovechando materia prima que actualmente es desecho (por ejemplo scrap de la industria de los cereales) y reconvertirlo en un material útil. A su vez, esta investigación puede sentar las bases para futuras investigación.

Palabras clave: Impresión 3d, Micelio de Hongos, Kombucha

\section{Abstract}

The first three industrial revolutions had the characteristic that they developed at times in history where natural resources were considered to be unlimited and that mass production would not have consequences on the environment and people. However, in recent years the serious impact that human activity has had on the environment has been demonstrated. This is why the foundations have been laid for the fourth industrial revolution, where a productive, social and economic model is proposed that changes the current system in an innovative, efficient and sustainable way.

The authors José Luis del Val (2016); Klaus Schwab (2016); Joao Saint-Aubyn (2016), among others, characterize the fourth industrial revolution or industry 4.0 as a new paradigm that aims to make a qualitative leap through the evolution and connection of tools and resources in search of forming the so-called 'Smart factories' . This model aims to give competitiveness to the industry through custom production and design. It seeks to link digital, physical and biological systems to optimize human and environmental well-being. It aims to direct production towards a circular economy, where the main objectives are to satisfy the needs of users and generate a paradigm shift in production, reducing waste and using renewable energy. It also accompanies the new circular economy model, which is interrelated with sustainability, whose main objective is to keep products, materials and resources in circulation for as long as possible, reducing waste production. It is about implementing a circular, non-linear system, based on the principle of closing the life cycle of products. Within this revolution, design plays a fundamental role in achieving the expected end. It is necessary to develop a designed system that allows the insertion of this revolution into the current environment, facilitating understanding and results. Biofabrication is incorporated into this circular scenario, which involves the application of biotechnology to cultivate and grow objects in the place of manufacture. This way of producing creates a paradigm shift, since bioproduction responds to the Circular Economy, where garbage does not exist and waste is recycled and transformed and, being biodegradable, returns to its origin.

It is in this new conception of manufacturing through the cultivation of materials where we position ourselves in research with the aim of finding solutions through design, biofabrication and the use of digital manufacturing technologies. For this, a work plan and use of methodologies are proposed to reach results through practical validation that will allow the introduction of an innovation to the current production system. It is proposed to generate designs that are introduced to the current system, replace plastic materials such as expanded polystyrene (tergopol) and collaborate with the environment under a circular economy model, that is, taking advantage of raw material that is currently waste (for example scrap from the cereal industry) and convert it into a useful material. In turn, this research can lay the foundation for future developments.

Keywords: 3d printing, Mushroom Mycelium, Kombucha 


\section{Introducción}

El presente trabajo tiene como fin comentar lo que se investigando para la realización del doctorado en Ingeniería en materiales.

La cuarta revolución industrial está relacionada con la implementación de la tecnología como la fabricación digital y la simulación 3D, principalmente en la Industria. A su vez esta revolución viene acompañada de un modelo de economía circular y todo un movimiento sociocultural que se está dando cuenta del daño que le hemos hecho al mundo generando residuos, generando contaminación lo que afecta al cambio climático y a los demás seres vivos como es el caso de los peces que están en un océano con toneladas de plásticos y micro plásticos. Esta revolución está llevando a un cambio en todos los niveles de la cadena productiva identificando que el diseño industrial cumple un rol fundamental a la hora de proponer innovaciones y cambios con resultados positivos, ya que el diseñador a través de diversas metodologías entre ellas design thinking, diseño centrado en las personas, etc. logra entender en profundidad los verdaderos problemas y puede proponer una solución efectiva.

Por otro lado está la biofabricación, la cual se define como la aplicación de la biotecnología para cultivar y hacer crecer los objetos en lugar de manufacturarlos. Este concepto y cambio en la manera de concebir la materia cambia la forma en que nos vinculamos con el medio ambiente. Estamos acostumbrados a extraer todo. Esta manera de producir crea un cambio de paradigma, ya que la bioproducción responde a la Economía Circular, en donde la basura no existe ya que los residuos se reciclan y se transforman y, al ser biodegradables, vuelven a su origen.

Finalmente estos materiales biofabricados pueden ser trabajados con tecnologías de fabricación digital las cuales permiten aprovechar todo el potencial de estos materiales para trabajar la forma y su materialidad y así optimizar el diseño para los requerimientos técnicos y para los esfuerzos que tenga que soportar la estructura, apoyándose de software de simulación y todo la tecnología que trae la cuarta revolución industrial.

Como base del trabajo consideramos necesario desarrollar un sistema de diseño que conjugue los instrumentos que ofrece éste nuevo modelo de industria circular (impresión y simulación 3D) trabajando con biofabricacion para lograr una alternativa de producción con materiales naturales y sustentables.

\section{Desarrollo}

El diseñador industrial está siempre relacionado con empresas, instituciones y por ende con procesos de innovación y los diseños que genera impactan en la sociedad y el medio ambiente. Esta conciencia social y el impacto en los procesos de cambio tecnológico, social y económico plantea una constante reflexión sobre las habilidades y herramientas que necesita el diseñador industrial para generar este impacto positivo.

Para mejorar la situación actual del medio ambiente se plantea un cambio en los modelos productivos, de un modelo lineal en donde todo lo que se produce se consume y posteriormente se desecha a un modelo circular en donde la materia que en el modelo lineal se desecha, en este se reinserta en el modelo productivos. Ya que la definición de desecho es todo lo que no tiene utilidad, podemos cambiar esta realidad, ya que si le encontramos utilidad deja de ser un desecho.

Para este cambio de modelos lineales a circulares se necesita incorporar Innovación y diseño al sistema productivo, entendiendo a la innovación como un cambio que genera impacto.

El proceso de cambio se puede plasmar en el diseño y desarrollo de nuevos productos, servicios y procesos hasta un cambio

en la estrategia de producción y comercialización de productos. Como casos de innovación en servicios podemos mencionar la 
plataforma Uber en donde se le dio una vuelta a lo que ya se conocía. Uber permitió hacer más simple y económico el servicio de transporte privado. Ya que no existía una plataforma similar para pedir este servicio y surgió a partir de detectar la poca eficiencia con la que contaba el transporte de taxi al momento solicitar un medio de transporte en el lugar deseado. Y el impacto puede ser medible y trazable desde distintos enfoques, económico, social, cultural, ambiental etc. Esto se relaciona con el concepto de Ezio Manzini de Innovación social.

Manzini (2008) define el trabajo del diseño como la "creación de puentes (bridges) entre la técnica y la sociedad". Entendiendo la técnica como el ámbito donde aparece la tecnología, la invención, y que puede surgir desde los laboratorios o desde los experimentos de calle. Es en esta conexión de la técnica con las personas es que aparece la innovación. "El término innovación social hace referencia a los cambios en la forma de actuar de individuos o comunidades a la hora de resolver un problema y crear nuevas oportunidades. Estas innovaciones son producidas más por cambios de comportamiento que por cambios tecnológicos o económicos, y normalmente surgen de procesos participativos en lugar de jerárquicos." Manzini (2008).

Entendiendo que la innovación sucede cuando la tecnología se incorpora a la sociedad, y ella la incorpora. No obstante tenemos innovaciones tecnológicas muy rápidas, las cuales son un potencial tecnológico que todavía no se han aprovechado. Buonfino y Hilder (2010) mencionan que se hacen visibles cada vez más los problemas sociales y medioambientales, por lo que se puede predecir una gran oleada de innovación social. Esta innovación social podría dar un salto hacia la sostenibilidad, entendiéndola como una característica fundamental del desarrollo que asegura las necesidades del presente sin comprometer las necesidades de futuras generaciones.

Para generar un aporte a la innovación social en busca de la sostenibilidad se va a trabajar en el desarrollo de una nueva forma de producción: La biofabricación, que se define como la aplicación de la biotecnología para cultivar y hacer crecer los objetos en lugar de manufacturarlos. Esta manera de producir crea un cambio de paradigma, responde a la Economía Circular, en donde la basura no existe ya que los residuos se reciclan y se transforman y, al ser biodegradables, vuelven a su origen. El concepto de biofabricación, es decir, el de cultivo de objetos se está aplicando a través de diferentes prácticas experimentales combinando materiales para producir objetos naturales como es el caso de Suzanne Lee, diseñadora de moda con sede en Brooklyn, Nueva York; trabaja en la moda y con las tecnologías del futuro a través del cultivo de ropa. (Lee ,2012). Otro caso es el de Neri Oxman, arquitecta, diseñadora y profesora estadounidense-israelí en el MIT Media Lab, dirige el grupo de investigación Mediated Matter. En su trabajo combina el diseño, la biología, la informática y la ingeniería de materiales. Entre sus trabajos podemos mencionar el pabellón de seda, hilado por gusanos de seda liberados en un marco de nylon, un trabajo colaborativo entre seres vivos y brazos robóticos (Oxman , Kayser, Laucks y Firstenberg, 2013); la silla monocasco bioimpresa en 3D realizada con materiales que presentan distintos grados de elasticidad. (Oxman, 2014). También se puede mencionar a la empresa Ecovative Design LLC radicada en Nueva York, pionera en el desarrollo de biomateriales a base de micelio de hongos. Esta empresa logró desarrollar un ladrillo para la construcción con este material. (Kim y Ruedy, 2016).

Interesa trabajar el cruce entre la biofabricación y las nuevas tecnologías de fabricación digital, ya que creemos que es la tecnología que permite prototipar validar e innovar a bajo costo pero con gran impacto.

Dentro de las tecnologías de fabricación digital se propone utilizar la tecnología de impresión 3d, debido a que es la más versátil y económica y consideramos que es la más adecuada para procesar este tipo de materiales en forma de pastas. La tecnología de impresión 3d de tecnología FDM (deposición de material fundido) permite llevar un modelo digital a la realidad a través de la superposición de capas de material. Las más difundidas son las de impresión de filamento plástico, pero en nuestro caso lo que utilizaríamos es pasta de composites de biomateriales para a través de ella realizar diferentes pruebas de objetos 3D. La tecnología de impresión 3D no es nueva pero con la caída de la patente y la globalización la misma está teniendo un auge en todo el mundo por sus características únicas y su gran capacidad de innovación. Dentro de las características se resalta la capacidad de descentralizar la producción, la personalización y el bajo costo. La descentralización de la producción ya no es 
necesario mandar a producir a grandes industrias o a China y luego importar los productos. Teniendo un modelo $3 \mathrm{~d}$ se puede imprimir en cualquier lugar del mundo, dismuniyendo costos y tiempos. La personalización ya que permite la realización de productos a medida, permitiendo que productos como prótesis que suelen ser costosos y no totalmente a medida por la limitación de los procesos productivos tradicionales, con la impresión3d estas prótesis pueden ser totalmente personalizadas y de muy bajo costo. Finalmente el bajo costo debido las impresoras son de código abierto y hay toda una Cultura y Comunidad Maker, lo que genera que los miembros de la comunidad vayan haciendo mejoras a las impresoras que impactan en que sean más eficientes y económicas. Lo mismo ocurre con los proyectos, ya que hay muchos proyectos de innvoción abiertos que se van retroalimentando con el aporte de cada uno de los miembros de la comunidad.

El termino de cultura maker surge como una evolución del Do It Yourself (DIY), Hágalo usted mismo, vinculado con la tecnología. Movimiento que surge en los años 50 que engloba multitud de actividades y que incentiva al desarrollo de proyectos por uno mismo, ahorrando dinero, se entretiene y como una forma de entretenimiento.

La comunidad maker está integrada por personas que les gusta hacer cosas, es español seria fabricantes y que se apoyan de las herramientas tradicionales y de las nuevas tecnologías, entre ellas la impresora 3D para generar proyectos. A través del open data y la tecnología, los makers comparten con la propia comunidad su experiencia para que los demás puedan recrear los productos, sin necesidad de grandes inversiones de dinero y sin recurrir a las empresas para conseguirlo. Innovación y compartir son los fundamentos de esta comunidad.

\section{METODOLOGIA}

El desarrollo de este plan de doctorado involucra una fuerte incidencia de la parte experimental que se llevará a cabo a través de etapas consecutivas. El trabajo experimental necesario para cumplir con los objetivos planteados inicialmente será realizado en el marco de un grupo de investigación multidisciplinario, en el cual se aprovechará la experiencia y los conocimientos de directora y codirectora para guiar las actividades. A partir de los datos experimentales obtenidos, la postulante llevará a cabo el procesamiento de los mismos y la interpretación de los resultados obtenidos mediante las siguientes actividades y metodología:

Actividad 1: Búsqueda y actualización bibliográfica con el objetivo de actualizar y profundizar, durante todo el desarrollo del plan de trabajo. Se cuenta con el servicio de la biblioteca electrónica de la SECyT.

\section{Actividad 2: Preparación de situaciones y objetos de experimentación}

Se realizará un estudio de las herramientas y técnicas de biofabricación y de fabricación avanzada. En base a los resultados de este estudio se definirán las herramientas y procesos a utilizar. Se desarrollarán prácticas y ensayos con dichas herramientas y materiales del reino Fungi.

En paralelo, se realizarán cursos de software de modelado 3D (Rhinoceros, Grasshopper, Autodesk Within, Fusion 360) como así también de software de configuración de archivos para fabricación digital (Slic3r, Cura, etc.) y se llevarán a cabo ejercicios de diseño utilizando dichos softwares para desarrollar moldes y formas que se utilizarán en el procesamiento de biomateriales.

Se buscarán las materias primas más adecuadas para las pruebas y ensayos, combinando hongos con sustratos orgánicos provenientes de descartes de la agroindustria (aserrín, rastrojo, yeso, etc.).

Se realizarán ensayos, pruebas y experimentos de biocultivos con las formas diseñadas. Se caracterizarán los sustratos a utilizar (contenido de humedad, composición elemental, densidad, etc.).

\section{Actividad 3: Diseño y preparación de Biomateriales}

En relación a los resultados obtenidos en los ensayos se elaborarán ejercicios prácticos de diseño con implementaciones en la industria y en productos. Se analizarán las situaciones productivas convencionales y no convencionales con los diseños propuestos. Se caracterizarán los biomateriales previamente diseñados en el laboratorio para analizar en detalle la influencia del tipo de sustrato utilizado. Se evaluarán la rigidez y modulo dinámico, resistencia a la fatiga, resistencia al deslizamiento y adherencia estática entre capas (Según Normas IRAM). En esta parte, también se determinarán las limitaciones de uso que puede tener a mayor escala. Específicamente se llevarán a cabo dos tipos de estudio:

a) Estudio de viabilidad técnica de la tecnología: se seleccionarán algunas formulaciones y serán implementadas en situaciones reales. 
b) Evaluación ambiental de la tecnología: se realizará a través de una Evaluación de Ciclo de Vida siguiendo la metodología recomendada por la norma internacional ISO 14040-43. El impacto ambiental de los productos se comparará con el impacto causado por los productos convencionales.

Los ensayos se realizarán según normas IRAM en los casos que sea posible. No se descarta la posibilidad de utilizar otras normas internacionales para realizar otros ensayos que puedan surgir durante la ejecución del plan de trabajo.

\section{Actividad 4: Optimizar los parámetros}

Se deberán optimizar los parámetros para obtener prototipos que se ajusten a los requerimientos técnicos planteados, principalmente en lo que se refiere a ensayos mecánicos normalizados de acuerdo al uso. En base a los resultados obtenidos se realizará una estimación de la viabilidad económica de la tecnología.

\section{Actividad 5: Conclusión y comunicación de resultados}

Discusión de los resultados y selección de los procesos. Conclusiones y redacción de la tesis. Se comunicarán los resultados en congresos y se publicarán en Revistas Científicas Indexadas.

El trabajo de investigación inicio en abril 2020 por lo cual en estos primeros meses se avanzo con la Búsqueda y actualización bibliográfica recopilando antecedentes locales e internacionales. También se comenzó con el diseño de la impresora de pastas y se están realizando las primeras pruebas de los mecanismos del dispositivo de impresión. Esta impresora permitirá realizar piezas con los biomateriales, permitiéndonos explorar la flexibilidad de la forma y las posibles materialidades.

\section{Conclusiones}

La pandemia impacto en el contexto y en el desarrollo de proyectos. No obstante creo que esta nueva normalidad nos hace reflexionar sobre la vulnerabilidad de la sociedad y también su capacidad para reinventarse y reaccionar ante los cambios y las amenazas.

Queda mucho por hacer y esta investigación recién está en una primera etapa de aproximación al campo de estudio pero considero que puede generar buenos resultados y que los mismos pueden ser aplicados para generar innovación a través del cambio en el sistema productivo y un impacto positivo a nivel social, económico y medioambiental.

\section{Referencias}

Manzini, E. (2008). Cuando todos diseñan. Milán, Italia. Experimenta

Manzini, E., Jégou , F. (2008). Servicios Participativos. Diseño e innovación social para la sostenibilidad. Revista Experimenta 63. Recuperado de: https://www.experimenta.es/noticias/grafica-y-comunicacion/servicios-participativosdiseno-innovacion-social-sostenible/

Buonfino, A, Hilder, P. (2010) Neighbouring in contemporary Britain. Young Foundation. Recuperado de: https://youngfoundation.org/wp-content/uploads/2013/06/Neighbouring_in_contemporary_Britain.pdf

Lee, S. (2012) Grow your own clothes. Recuperado de: http://www.ted.com/talks/lang/en/suzanne_lee_grow_your_own_clothes.html

Oxman N., Kayser N., Laucks J., Firstenberg M. (2013) Robotically Controlled Fiber-based Manufacturing as Case Study for Biomimetic Digital Fabrication. Massachusetts Institute of Technology, Cambridge, Massachusetts, USA. Taylor \& Francis, recuperado de: https://neri.media.mit.edu/assets/pdf/1__SIM123_Robotically_Controlled_Fiberbased_Manufacturing_as_Case_Study_for_Biomimetic_Fabrication.pdf

Oxman, D. Dikovski, B. Belocon, W. C. Carter, (2014) Gemini: Engaging Experiential and Feature Scales through MultiMaterial Digital Design Fabrication N. Journal of 3D Printing and Additive Manufacturing, recuperado de: https://neri.media.mit.edu/publications.html

Kim, Y., Ruedy, D. (2016). Mushroom Packages An Ecovative Approach in Packaging Industry. Handbook of Engaged Sustainability Recuperado de https://www.researchgate.net/publication/336146038_Mushroom_Packages_An_Ecovative_Approach_in_Packaging_Ind ustry 